\title{
Oral lichen planus involving the palate: Case report and review of literature
}

Shaheen Ali Ahmed ${ }^{(1)}$; Ali Fakhree Alzubaidee ${ }^{(2)}$

\begin{abstract}
Lichen planus is a mucocutaneous disease affecting approximately 1.5 to $2 \%$ of the world population. Women are most of ten affected and lichen planus is usually reported in individuals over 50 years of?age The etiology? ? lichen planus is unknown at present and the disorder has been classified as a chronic disease of immune-mediated pathogenesis. The oral lesions are usually bilateral and involve the buccal mucosa in about 90 per cent of all cases. In descending order of frequency the tongue, gingivae, alveolar ridge, lips, and less commonly the palate may also be affected. In this paper, we are presented a case , 65-year-old female patient who came to department of Oral and Maxillofacial Medicine in Khanzad Center with a chief complaint of burning sensation of the mouth especially hard palate, bilateral buccal mucosa and lower anterior gingiva for about 10 years duration, aggravated by hot and spicy food. The diagnosis was confirmed by biopsy as lichen planus. The skin was not involved lesion
\end{abstract}

Keywords: Oral lichen planus, Palatal lichen planus, Burning sensation, Buccal mucosa

\footnotetext{
${ }^{(1)}$ College of Dentistry /Hawler Medical University, shahenallany@yahoo.com.

${ }^{(2)}$ Kurdistan Board for Medical Specialists
}

\section{Introduction}

Lichen planus is considered as a chronic inflammatory disease affecting the skin and mucous membranes. Oral lichen planus (OLP) can occur in association with cutaneous lichen planus or alone. ${ }^{1,2}$ It is estimated that $50 \%$ to $70 \%$ of adult LP patients have both skin and oral lesions and approximately $25 \%$ of patients presented with oral lesions alone. ${ }^{3,4}$ It is seen worldwide, mostly in the fifth to sixth decades of life, and is twice as common in women as in men $^{5}$. The etiology of lichen planus remains uncertain, but many factors have been implicated. Such factors include genetic predisposition, infective agents, systemic diseases, graftversus-host disease (GVHD), drug reactions, vitamin deficiencies, and hypersensitivity to dental materials ${ }^{6}$. Lichen planus has been associated with several autoimmune diseases, including lupus erythematous, pemphigus, Sjögren's syndrome, and autoimmune liver disease ${ }^{7,8}$.

It is seen clinically as reticular, papular, plaque-like, erosive, atrophic or bullous types ${ }^{1,9,10,11}$. In practice most clinicians prefer 2 clinical designations: (1) reticular OLP; and (2) erosive OLP. Reticular OLP typically presents as asymptomatic white keratosis, while the erosive form is erythematous and frequently painful ${ }^{5,12}$. The most frequently involved oral sites include the mucosa of the cheek, tongue and gingiva. The mucosa of the palate and floor of the mouth is rarely affected 13-15. OLP is diagnosed clinically by means of a biopsy for histopathological analysis 16 . The classical microscopic features observed in the oral mucosa include hyperorthokeratosis or hyperparakeratosis, acanthosis, thickening of the spinous layer, liquefaction of the basal layer accompanied by the degeneration 
of keratocytes, and lymphocyte infiltration of the lamina propria ${ }^{16-18}$.

It is important for all clinicians to be aware of its clinical presentations and management, because OLP is one of the most common mucosal conditions affecting the oral cavity. The most widely accepted treatment for OLP is topical corticosteroids. Alternative treatments include retinoids, cyclosporine, tacrolimus, surgery, and carbon dioxide (CO2) laser ${ }^{1}$.

\section{Case Report}

A 65-year-old upper edentulous female patient who came to department of Oral and Maxillofacial Medicine in Khanzad Center with a chief complaint of burning sensation of the mouth especially hard palate, bilateral buccal mucosa and lower anterior gingiva for about 10 years duration, aggravated by hot and spicy food. She complained now of difficulty in having even soft foods. Dental history showed that she has had old denture for 20 years ago. Her medical and family histories were unremarkable and there was no history of any drug intake during the last 12 months. On examination, no cutaneous lesions were evident. On intraoral examination, there were erythematous red and white patches in the premaxilla extended to the premolar region (Figure 1), the re d erosive area with irregular white stria (reticular ) boarder on the right and left buccal mucosa (Figure 2), and red erosive lesion on the lower anterior region (desquamative ) gingivitis (figure 3). The lesions were non tender.

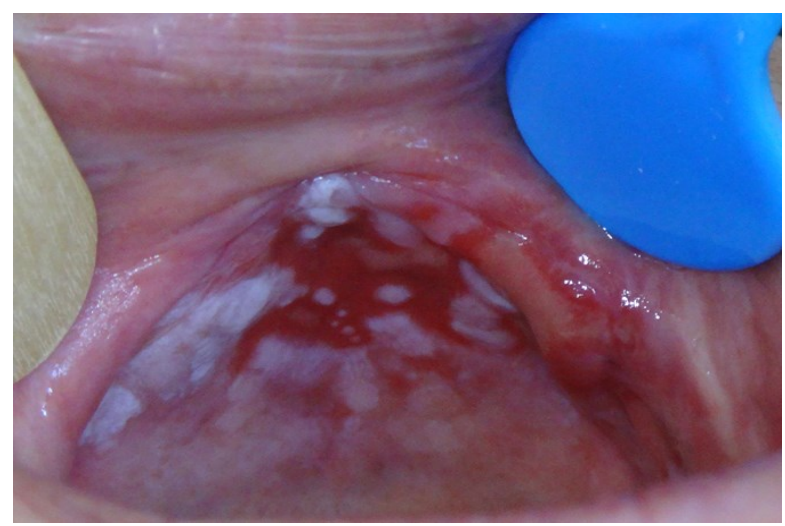

Figure 1: Red erythematous and white patches in premaxilla extended to the premolar area.

The patient was advised for biopsy. Routine hematological investigations and biochemistry (complete blood count and film, ESR, fasting blood sugar, renal function test, liver function test, and viral screening) yielded negative results. Tow incisional biopsies were done the palatal biopsy showed epithelial ulceration, dense neutrophilic and lymhpocytic infiltration, while the alveolar mucosa biopsy showed area of epithelial ulceration and a focal area of epithelium that showed hyperkeratosis, wedge shape hypergranulosis, irregular acanthosis and basal layer liquefaction. A lichenoid lymphocytic infiltration was seen in the subepithelium (Histopathological findings were consistent with a diagnosis of oral lichen planus.

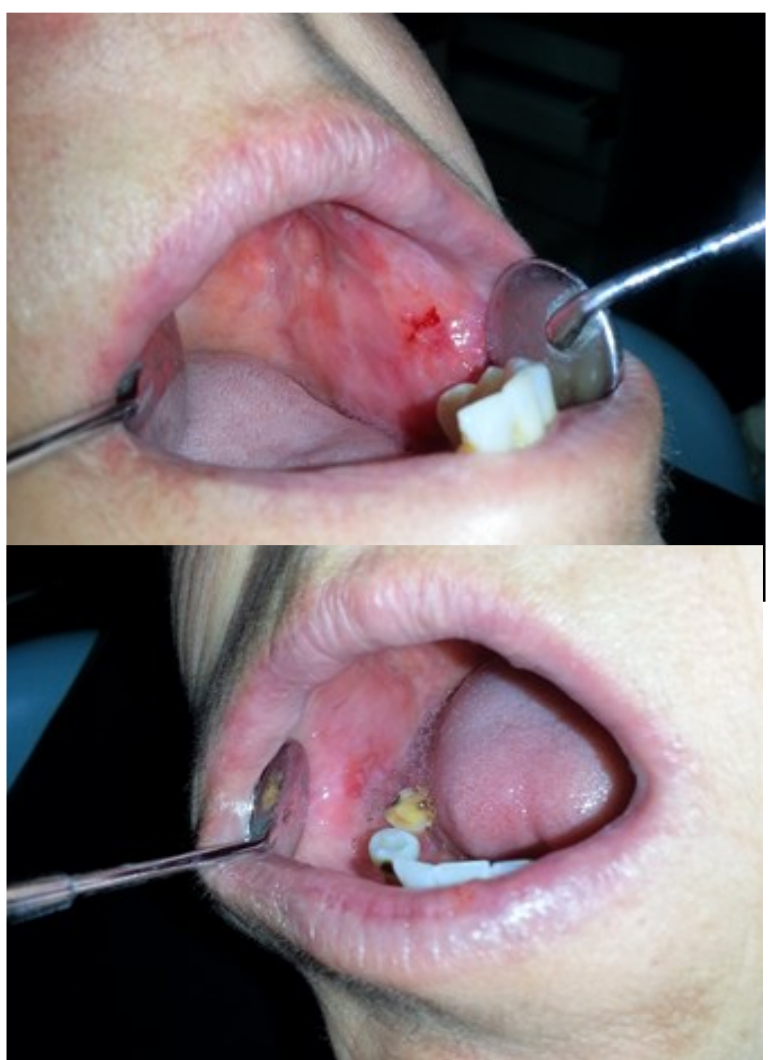

Figure 2: Bilateral erosive and reticular lesions on buccal mucosa.

The patient was educated and motivated regarding a proper plaque and denture care control regimen that included brushing thrice daily with a soft brush and tooth paste and rinsing twice daily with alcohol free chlorhexidine for 2 weeks. The patient was advised to avoid eating spicy foods while her lichen planus was active and to use topical corticosteroid cream containing 0.1 percent triamcinolone acetonide (Kenacort 
with systemic steroids. Initially, she was being reviewed weekly. The active lesions had improved at the first month of treatment, the dose were tapered and kept only under topical steroid she was being reviewed after every 1-month period (figur4).

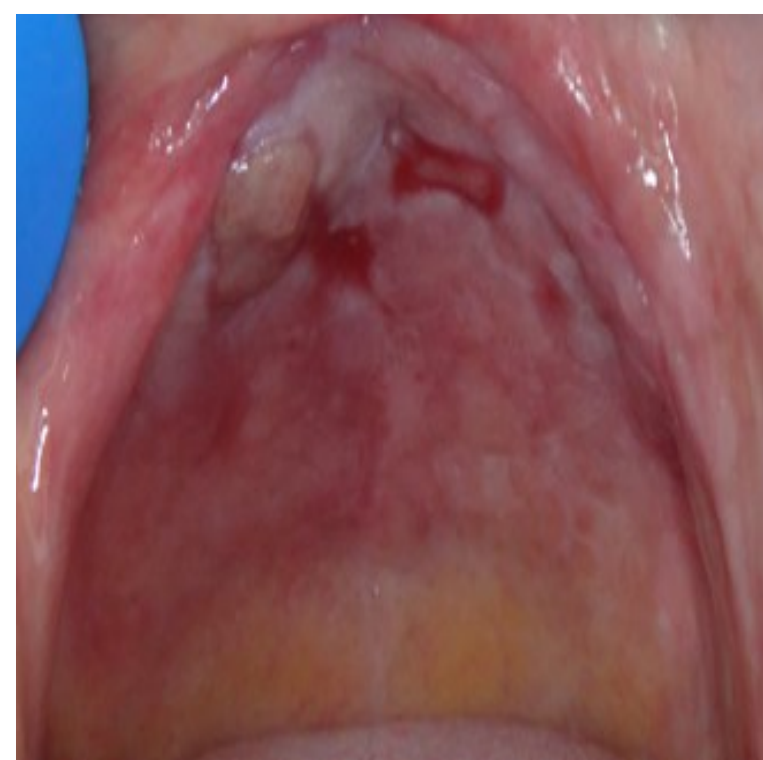

Figure 3: Desqumative gingivitis of lower anterior gingiva.

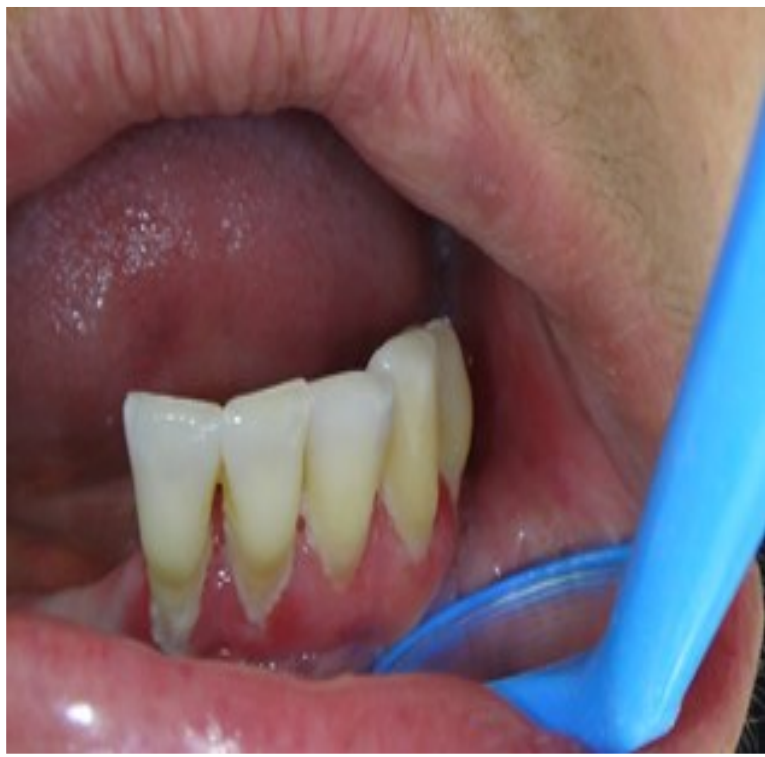

Figure 4: The lesions become improved after

Treatment.

\section{Discussion}

Oral lichen planus (OLP) can occur in association with cutaneous lichen planus or alone in our case the patient presented with out skin lesion only oral lesion. Absence of cutaneous involvement in the pre- sent case is not unusual considering that associated lesions are typically found in only approximately 15 percent of patients with OLP22. $1-2 \%$ of the population worldwide suffers from OLP. Female predilection was found with a male to female ratio of $1: 2$ especially among the middle aged 9, 20 .In our case the patient was in her sixth decade of life.

The different etiological factors considered for LP are genetic background, drugs, autoimmunity, immunodeficiency, stress, diabetes, hypertension, malignant neoplasm, and bowel disease. ${ }^{21}$ The various koebnerogenic factors are dental materials, an infectious agent such as human papillomavirus, food allergy, habits like lip chewing, and trauma from sharp cusps. ${ }^{11}$ In our patient the palatal lesion due to chronic irritation of old denture.

The buccal mucosa, lips, gingivae, floor of the mouth, and palate are usually affected, in a descending order of frequency ${ }^{16}$. The lesion is usually bilateral and symmetrical and may manifest in six different clinical forms mainly reticular, popular, plaque-like, erosive, atrophic and bullous form. The most commonly involved site is buccal mucosa, tongue and gingiva. ${ }^{23}$ Here, we emphasize occurrence in female patient lesion presented in the palate, buccal mucosa and lower anterior attached gingiva which is unusual.

The classical clinical presentation of the lesion is sufficient to make an accurate diagnosis $^{2}$. The oral biopsy of the lesion with histopathologic confirmation is recommended to confirm the clinical diagnosis and also to exclude chances of dysplasia and malignancy. Most cases of oral lichen planus are often asymptomatic but the atrophic and/or erosive forms cause varied degrees of discomfort, which prompts the search for early professional care. However, as in the present case, patients may have the disease for a long time because lichen planus is a chronic condition characterized by recurrent exacerbation and remission periods.

\section{Conclusion}

Classically the oral lichen planus found on the buccal mucosa and gingiva. This paper highlights the patient with oral lichen 
planus in the palate buccal mucoasa, and lower anterior gingiva. Topical steroids used alone or in combination with other immunomodulatory topical agents is a widely accepted first choice of relief in most patients. Prolonged use of systemic medications and elimination of the causative factor is essential to eradicate the disease.

\section{References}

1. Sugerman PB, Savage NW, Walsh $L$, Zhao ZZ, Zhou XJ, Khan A, et al. The pathogenesis of oral lichen planus. Crit Rev Oral Biol Med 2002; 13:350-65.

2. Patil A, Prasad S, Ashok L, Sujatha GP. Oral bullous lichen planus: Case report and review of management. Contemp Clin Dent 2012; 3:344-8.

3. Handa S, Sahoo B. Childhood lichen planus: a study of 87 cases. Int J Dermatol. 2002; 41:423-7.

4. Kumar V, Garg BR, Baruah MC, Vasireddi SS. Childhood lichen planus (LP). J Dermatol. 1993; 20:175-7.

5. Eisen D, Carrozzo M, Bagan Sebastian JV, Thongprasom K. NumberV oral lichen planus: clinical features and management. Oral Dis 2005; 11:338-49.

6. Soames JV, Southam JC. Oral Pathology. 3rd ed. Oxford: Oxford University Press; 1998:151 $-156$.

7. Scully C, Beyli M, Ferreiro MC, et al. Update on oral lichen planus: etiopathogenesis and management. Crit Rev Oral Biol Med. 1998;9 (1):86-122.

8. Alam F, Hamburger J. Oral mucosal lichen planus in children. Int J Paediatr Dent. 2001;11(3):209-214.

9. McCreary CE, McCartan BE. Clinical management of oral lichen planus. Br J Oral Maxillofac Surg 1999; 37:338-43.

10. Scully C, Carrozzo M. Oral mucosal disease: Lichen planus. Br J Oral Maxillofac Surg 2008; 46:15-21.
11. Gorouhi F, Davari P, Fazel N. Cutaneous and mucosal lichen planus: A comprehensive review of clinical subtypes, risk factors, diagnosis, and prognosis. Scientific World Journal 2014; 2014:742826

12. Laeijendecker R, Van Joost T, Tank B, Oranje AP, Neu- mann HA. Oral lichen planus in childhood. Pediatr ?Dermatol. 2005; 22:299-304.

13. Xue JL, Fan MW, Wang SZ, Chen XM, Li Y, Wang $\mathrm{L}$ : A clinical study of 674 patients with oral lichen planus in China. J Oral Pathol Med. 2005, 34: 467-72.

14. Eisen D. The clinical features, malignant potential, and systemic associations of oral lichen planus: a study of 723 patients. J Am Acad Dermatol. 2002, 46: 207-14.

15. Ingafou M, Leao JC, Porter SR, Scully C: Oral lichen planus: a retrospective study of 690 British patients. Oral Dis. 2006, 12: 463-8.

16. Ismail SB, Kumar SK, Zain RB. Oral lichen planus and lichenoid reactions: $E$ tiopathogenesis, diagnosis, management and malignant transformation. J Oral Sci 2007;49:89-106.

17. Anuradha $\mathrm{CH}$, Reddy BV, Nandan SR, Kumar SR: Oral lichen planus. A review. N Y State Dent J. 2008, 74: 66-8.

18. Mollaoglu N: Oral lichen planus: a review. $\mathrm{Br} \mathrm{J}$ Oral Maxillofac Surg. 2000, 38: 370-7.

19. Greenberg MS, Glick M, Ship JA. Burkets Oral Medicine. 11th ed. Hamilton: BC Decker Inc. Publication; 2008.

20. Rajendran R. Oral lichen planus. J Oral Maxillofac Pathol 2005;9:3-5.

21. Roopashree MR, Gondhalekar RV, Shashikanth MC, George J, Thippeswamy SH, Shukla A. athogenesis of oral lichen planus - A review. J Oral Pathol Med 2010; 39:729-34.

22. Eisen D. The evaluation of cutaneous, genital scalp, nail, esophageal, and ocular involvement in patients with oral lichen planus. Oral Surg Oral Med Oral Pathol Oral Radiol Endod 1999; 88:431-6.

23. Silverman S Jr. Oral lichen planus: A potentially premalignant lesion. J Oral Maxillofac Surg 2000; 58:1286-8. 